\title{
COMUNICACIÓN
}

\section{ESTUDIO HEMATOLÓGICO COMPARATIVO DEL SAJINO (Tayassu tajacu) CRIADO EN CAUTIVERIO EN LIMA E IQUITOS}

\author{
Miluska Navarrete Z.', Olga Li E. ${ }^{2}$, Enrique Montoya G. ${ }^{3}$ y Hugo Gálvez C. ${ }^{3}$
}

\section{Abstract}

Hematological values for Peruvian peccaries (Taycussu iujacu) are reported. Blood samples were taken from 11 animals at Parque de las Leyendas Zoo in Lima, 7 at the Biodiversidad Amazónica (BIOAM) breeding center in Iquitos and 4 at the Centro Piloto para Crianza del Majaz (Universidad Nacional de la Amazonia Peruana) also in lquitos. Following sedation with ketamine $(12 \mathrm{mg} / \mathrm{kg})$ using a pole syringe or cervate, blood samples were collected from the cephalic vein using sterile tubes containing EDTA and blood smears made. Hematological analysis was carried out at the Clinical Pathology Laboratory, Faculty of Veterinary Medicine, San Marcos University, in Lima, yielding the following results for the Lima and lquitos peccaries respectively: $\mathrm{Hb}$ (g/dl) $15.48 \pm \mathrm{l} .60$, II.26 $\pm 0.98 ; \mathrm{Ht}(\%) 35.09 \pm 4.41,32.64 \pm 5.35$; erythrocytes (x10\%/ul) $6.036 \pm 1.58,3.30 \pm 0.99$; leukocytes $\left(\mathrm{x} 10^{3} / \mathrm{ul}\right) 13.77 \pm 4.85,15.29 \pm 6.69$; band neutrophils $(\%) 1.33 \pm 0.58,1.6 \pm 0.55$; seginented neutrophils (\%) 48.27 $\pm 7.2,23.64 \pm 12.51$; lymphocytes (\%) 50.64 56.96 , $69.09 \pm 11.23$; eosinophils (\%) $1.33 \pm 0.52,6.09 \pm 3.24$; monocytes $(\%) 0,1$; basophils $(\%) 0$, 1; platelets (thrombocytes/ml) $338220 \pm 221760$ and $167490 \pm 54390$. The hematological values of the Lima peccaries are similar to those published for North American animals, but differ from the lquitos results which contained a high percentage of lymphocytes and eosinophiles reflecting anemia.

Key words: Peccary (Tayassu rajacu), hematological values.

Palabras clave: Sajino (Tayassu tajacu), valores hematológicos.

En el Perú, la investigación en fauna silvestre se ha incrementado en los últimos años. El sajino (Tuyassu tajacu) en la Región Amazónica se cría para la producción de la llamada "carne de monte" y para la exportación de pieles muy cotizadas en el extranjero. En la Costa, la finalidad de su crianza es recreacional y educativa. En nuestro medio, no hay reportes sobre sus valores hematológicos, cuyo conocimiento es básico para el diagnóstico de enfernedades infecciosas, parasitarias, etc. La literatura sólo

\footnotetext{
- Práclica Privada

"Laboratorio de Patologia (Vinica - FMI" - ('NMASA).

E-mail: dl 7(1)23 (üunmsm.edu.pe

${ }^{3}$ EE IITTA - Iquitos. Email: ivitaiquigterracom pe
}

reporta valores hematológicos que datan de la década de los 70 realizados en zoológicos de Norteamérica, pero en un pequeño número de animales y sin especificar sexo ni edad.

El presente trabajo se realizó con el objetivo de conocer los valores hematológicos de hemoglobina. hematocrito, conteo de eritrocitos, leucocitos, plaquetas y recuento diferencial de leucocitos de esta especie silvestre con la finalidad de ayudar a veterinarios del zoológico en la evaluación sanitaria de esta especie.

Las muestras fueron obtenidas de sajinos del Zoológico Patronato Parque Las Leyendas (PATPAL) de Lima. en el 
Zoocriadero Biodis ersidad Amazónica S.R.L (BIOAM) y en el Centro Piloto de Crianza del Majaz de la ('niversidad Nacional de la Amazonía Peruana (UNAP) en lquitos. durante los meses de agosto y setiembre de 1999.

Se muestrearon en total 22 sajinos distribuidos como siguc: 11 sajinos (6 hembras y 5 machos adultos) de PATPAL, los cuales recibieron constante atención médica y alimentación balanceada: 7 sajinos ( 6 machos y 1 hembra subadultos) de BIOA.M y 4 sajinos (machos juveniles) de la (INAP. los que sólo recibieron atención a heridas superficiales y la alimentación se basó en yuca. frutas de la zona! molluelo de soya.

Los sajinos, prev io ayuno, fueron tranquilizados con clorhidrato de hetamina en dosis de $12 \mathrm{mg} / \mathrm{kg}$ de peso vivo via intramuscular, utilizando bastón jeringa o cerbatana.

Las muestras de sangre se obtuvieron de la vena cefálica en tubos de ensayo con anticoagulante EDTA, previamente rotulados; asi mismo se hicieron frotices sanguineos en láminas portaobjeto.

Los tubos rotulados se trasladaron en cajas térmicas con bolsas refrigerantes al Laboratorio de Patología Clínica de la Facultad de Medicina Veterinaria de la Universidad Nacional Mayor de San Mareos, para su procesamiento.
Las muestras se procesaron según el Manual de Patologia Clínica en Veterinaria de Benjamin ( 1991), utilizando los siguientes procedimientos:

- Para Hematocrito: Método del Microhematocrito (\%).

- Para Hemoglobina: Método de la Cianometahemoglobina (g/dl).

- Para Recuento Globular: Eritrocitos (x $10 /$ ul) y Leucocitos $\left(x 10^{3} / u 1\right)$.

- Para Recuento Diferencial : Tinción Wrigth $(\%)$

- Para Recuento Plaquetario: Método Indirecto (trombocitos/ul).

Se empleó la media aritmética y la desviación estaindar para analizar los resultados.

\section{Serie Eritrocitica:}

En el Cuadro 1, se observa que los valores de la serie critrocítica encontrados en Iquitos están disminuidos en comparación con los animales criados en Lima, que reflejan un cuadro anćmico en estos animakes; observándose anisocitosis, poicuulocitosis e hipocromía.

La anemia puede deberse a que los sajinos en Iquitos reciben una dieta como herbivoros fructivoros, a diferencia de los sajinos criados en Lima que reciben una alimentación más completa.

Cuadro 1. Valores medios de la serie eritrocítica para sajinos (Turassu tajacu) muestreados en Lima e Iquitos.

\begin{tabular}{lcccc}
\hline \multirow{2}{*}{ Serie critrocitica } & \multicolumn{2}{c}{ Lima } & \multicolumn{2}{c}{ Iquitos } \\
\cline { 2 - 5 } & Media & D.E. & Media & D.E. \\
\hline Ilemoglobina $(\mathrm{g} / \mathrm{dl})$ & 15.48 & 1.60 & 11.26 & 0.98 \\
Hematocrito $(\%)$ & 35.09 & 4.41 & 32.64 & 5.35 \\
Eritrocitos $\left(\mathrm{x} 10^{\prime} / \mathrm{ul}\right)$ & 6.36 & 1.58 & 3.30 & 0.99 \\
Pilas globulares & $* * *$ & & $* *$ & \\
\hline
\end{tabular}


La presencia de pilas globulares fue muy notoria en los frotices sanguíneos de los sajinos muestreados en Lima, mientras que fue escaso en los sajinos muestreados en Iquitos debido al mismo cuadro anémico por la lisis de eritrocitos.

\section{Serie Leucocítica:}

Los valores de leucocitos se muestran en el Cuadro 2. Los sajinos muestreados en lquitos muestran un mayor porcentaje y mayor variación, ésto puede deberse a que son animales más jóvenes (Doxey, 1987) y sujetos a mayor estrés de captura, pues necesitaron ser capturados con redes y tranquilizados usando cerbatana. El estrés por manejo influye en la presentación de leucocitosis físiológica.

De los resultados hematológicos de la serie eritrocítica y leucocítica encontrados en un total de 22 sajinos (Tayassu tajacu) muestreados en Lima e lquitos; los valores hematológicos reportados en Lima, se asemejan a los reportados en Norteamérica y son considerados normales, mientras que los valores hematológicos reportados en lquitos tienden a estar disminuidos en la serie eritrocítica (anemia) e incrementados en la serie leucocítica (linfocitosis y eosinofilia) debido posiblemente a que son animales jóvenes e influenciados por factores como la alimentación y la presencia de hemoparásitos.

Cuadro 2. Valores medios de la serie leucocítica para sajinos (Tayassu tajac'u) muestreados en Lima e Iquitos.

\begin{tabular}{lcccc}
\hline \multicolumn{1}{c}{ Serie Leucocítica } & \multicolumn{2}{c}{ Lima } & \multicolumn{2}{c}{ Iquitos } \\
& Media & D.E. & Media & D.E. \\
\hline Leucocitos (x 10\%/ul) & 13.77 & 4.85 & 15.29 & 6.69 \\
Neutrófilos: & & & & \\
\multicolumn{1}{c}{ Abastonados (\%) } & 1.33 & 0.58 & 1.60 & 0.55 \\
$\quad$ Segmentados (\%) & 48.27 & 7.20 & 23.64 & 12.51 \\
Linfocitos (\%) & 50.64 & 6.96 & 69.09 & 11.23 \\
Eosinófilos (\%) & 1.33 & 0.52 & 6.09 & 3.24 \\
Monocitos (\%) & 0 & - & 1 & - \\
Basófilos (\%) & 0 & $-\overline{17}$ & 1 & \\
Plaquetas (trombocitos/ul) & 338220 & 221760 & & 54390 \\
& & & &
\end{tabular}

\section{Literaturn Citada}

1. Benjamin, M. 1991. Manual de patología clínica en Veterinaria. 3era Ed. p 9 - 129. Editorial LIMUSA. México.
2. Doxey, D.L. 1987. Patología clínica y procedimientos de diagnóstico en Veterinaria. 2da Ed., p 185-194. Editorial El Manual Moderno S.A. México. 